\title{
Utber einen Fall von bisher noch nicht beschriebener Paren- domyces-Erkrankung, die unter dem Bilde der tiefen (Sycosis parasitaria) und oberflächlichen Trichophytie verlief.
}

\author{
Von \\ Dr. M. Rischin, \\ Assistent der Klinik. \\ Mit 9 Textabbildungen. \\ (Eingegangen am 2\%. November 1920.)
}

Nachstehend möchten wir über eine Hautaffektion berichten, die wegen der Schwierigkeit ihrer diagnostisehen Sicherstellung, wegen des Verlaufes der Krankheit und der Therapie unsere Aufmerksamkeit auf sich lenkte.

Anamnese. Pat. Z. F., Schweizer, aus dem Kanton Bern, 43 Jahre alt, Melker, will mit Ausnahme eines vor 7 Jahren durchgemachten Gelenkrheumatismus stets gesund gewesen sein. Speziell weiß er nichts von früheren Haut- oder Geschlechtskrankheiten. Z. war nie im Auslande. Die jetzt bestehende Hautaffektion datiert seit Anfang Januar 1919 (ungefähr 1 Monat vor Spitaleintritt). Zuerst trat eine kleine Efflorescenz in der Gegend des linken Handgelenks auf, ca. 8 Tage darauf an der linken Halsseite eine zweite Efflorescenz, die sich innerhalb weniger Tage über die ganze Bartgegend ausbreitete. Nachher entstand ein dritter Herd am rechten Vorderarm, dann ein vierter an der linken Brustseite. Subjektiv empfand der Mann ein Jästiges Brenngefühl an den erkrankten Stellen. In der Familie ist eine ähnliche Hautaffektion nicht bekannt. Z. meint, er habe sich das Leiden durch Übertragung einer ähnlichen Affektion bei seinen 5 Kälbern zugezogen. Spitaleintritt: 3. II. 1919. Mittelgroßer, kräftiger Mann. Innere Organe o. B. Urin und Temperatur normal.

Hautstatus. Die gesamte Bartgegend und die Haut des Halses und des Nackens zeigen krankhafte Veränderungen, ganz besonders an der linken Seite: auf geröteter Basis liegen mehr oder weniger stark elevierte, tief infiltrierte, rund. liche oder ovale, meistens konfluierende, ca. $1-1 \frac{1}{2} \mathrm{~cm}$ über das Hautniveau erhabene, geschwulstartige Knoten ron eher weicher Konsistenz, die an verschiedenen Stellen spontan, besonders reichlich jedoch auf Druck aus feinen Öffnungen ein übelriechendes, bräunlichgelbes Sekret entleeren. Der Bart ist im ganzen noch ziemlich gut erhalten, indes lassen sich die Haare an den stark alterierten Stellen mit der Pinzette leicht epilieren. Eigentlich geschwürige Prozesse bestehen nirgends. Die Abgrenzung gegen das umgebende normale Gewebe ist nicht sehr scharf, vielmehr bestehen teils runde, teils ovale, rötlich verfärbte Herde mit zentraler und peripherer Schuppung, ganz besonders im Nacken. Weiter unten über beiden 
Claviculae und deren Umgebung, sowie um die Mamilla links, am Rücken in der oberen und mittleren Partie, am linken Vorderarm, besonders an der Beugeseite und in der Ellenbogengegend, am rechten Vorderarm, an der Beugeseite desselben ïber dem Handgelenk (ein einzelner, fünffrankstiickgroßer Herd), am Abdomen unter dem Umbilicus sind überall runde oder ovale Herde zu finden mit geröteter, leicht infiltrierter Basis und reichlicher Schuppenbildung. Blasen sind nirgends vorhanden. Die übrige Haut und der behaarte Kopf sind frei von irgendwelcher krankhafter Veränderung. Drüsen: nicht vergrößert. Schleimhäute: normal. Nägel: o. B.

Sowohl das klinische Bild wie die Anamnese sprachen für. Trichophytia profunda der Bartgegend (Sycosis parasitaria) und Trich. superfic. et profunda am übrigen Körper. Pilze sind trotz mehrmaliger und sorgfältiger Untersuchung nie mit Sicherheit gefunden worden. Von Kulturen und verschiedenen angestellten Reaktionen wird unten die Rede sein.

Therapie. Umschläge mit Liq. alum. acet. sol. und Leinsamenkataplasmen in der Bartgegend, an den übrigen affizierten Stellen Chrysarobin $1: 2000$ in Zinkpaste.

7. II. 1919. Starke Suppuration in der affizierten Bartgegend. Temperatur: abends $38,1-38,3$, morgens $37,2-37,4$; jedoch ohne subjektive Erscheinungen. I3. II. 1919. Die Suppuration in der Bartgegend dauert an. Temperatur: unregelmäBig, subfebril. 17. II. 1919. Öfters

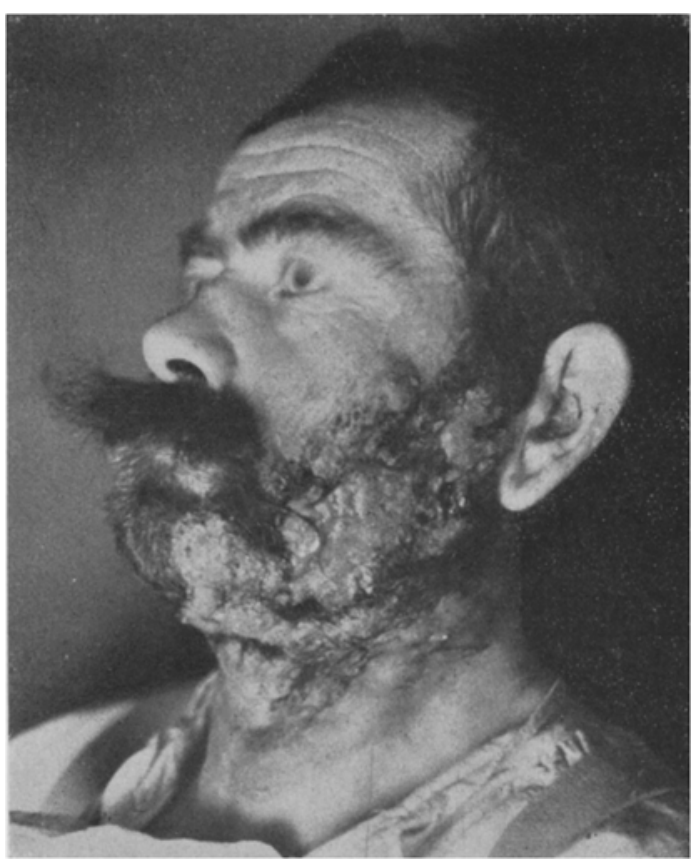

Abb. 1. Vor der Behandlung.

Konstipation, stets starke Suppuration. Temperatur: unregelmäßig. 27. II. 1919. Keine Änderung eingetreten. 9. III. 1919. Die Eiterung ist etwas zurückgegangen, immer noch leichtes Fieber am Abend. Das Allgemeinbefinden stets gut. 13. III. 1919. Röntgenbestrahlung. Abendtemperatur 38,7 (wahrscheinlich eine Reaktion auf die Röntgenbehandlung). 24. III. 1919. In der Bartgegend keine Veränderung. Die Herde an den anderen Stellen vollständig verschwunden. Da die bisherige Behandlung, die sich auf iber 6 Wochen ausdehnte, nicht den geringsten Erfolg gezeitigt hatte, verordnete mein Chef, Herr Prof. Naegeli, versuchsweise Jodkali intern 3 mal täglich 0,5. 3. IV. 1919. Die Affektion am Bart zeigt daraufhin eine auffallende Heilungstendenz. Die Sekretion ist wesentlich zu. rückgegangen. Jodkali wird von dem Patienten ohne jegliche Störung vertragen. Temperatur normal. Wir beginnen mit Jodkali bis auf $3 \mathrm{~g}$ pro die zu steigen. 20. IV. 1919. Die entzündliche Schwellung hat abgenommen, die Tumoren sind flacher und kleiner geworden, keine Eiterung. 10. V. 1919. Röntgenbehandlung. 
Keine Reaktion. Pat. wird entlassen. In der Bartgegend sind nur noch vereinzelte wulstige Erhabenheiten vorhanden. Pat. bekommt nach Hause die Ordination Jodkali 10:300 3 mal täglich I Eßlöffel. Er stellte sich auf unsere Einladung am 20. X. 1919 wieder vor. Es sind nur noch leicht erhabene, radiär verlaufende Narben zurückgeblieben (siehe Abb. 1 und 2).

Wie bereits erwähnt, wurde die Affektion der Bartgegend als Sycosis parasitaria und das Exanthem an der unbehaarten Haut als Trichophytia superficialis et profunda angesprochen. Die Sicherung der Diagnose mußte je-

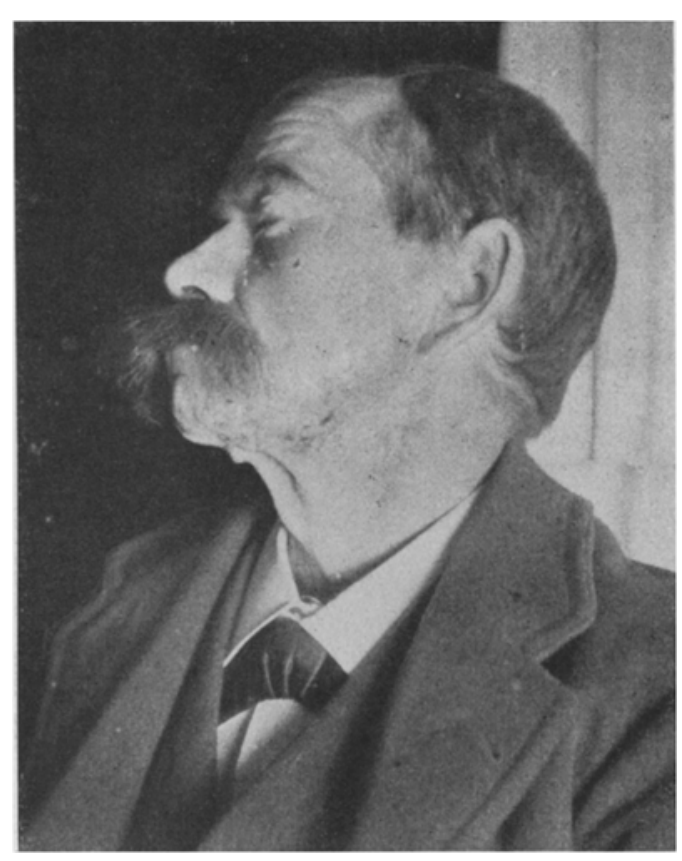

Abb.-2. Nach der Behandlung. doch den Laboratoriumsmethoden vorbehalten bleiben. Dieselben ergaben folgendes:

1. Pilze sind weder in den Haaren noch in den Schuppen gefunden worden. 2. In den $\mathrm{Ab}-$ strichpräparaten keine Drusen. 3. Die Färbung des Sekretesauf Tuberke]bacillen ergab ein negatives Resultat. Andere Formelemente, außer zahlreichen Leukocyten, sind nicht vorhanden. 4. Tuberkulinreaktionen: negativ. 5. Wa.R.: negativ. 6. Trichophytinreaktionen: (Tr. gyps., Achor. Quinck. und Microsp. Audouini): percutan negativ, intradermal positiv. 7. 'Sporotrichinreaktion: pereutan und intradermal negativ. 8. Verimpfung der Haare und Schuppen auf zuckerhaltige Nährböden (Maltose- und Glykoseagar) ergab ein negatives Resultat. Dagegen entwickelte sich auf den gleichen mit Eiter beschickten Nährböden nach 8 Tagen bei Zimmertemperatur in einem Reagensgläschen an einer Stelle eine verunreinigte, auf Pilze verdächtige Kolonie. Erst die mehrmalige Überimpfung dieser verdächtigen Stelle ergab später reine Pilzkolonien auf verschiedenen Nährböden (festen und flüssigen). In Ausstrichpräparaten dieser Kolonien waren rundliche oder ovale, meist ovoide, scharf konturierte, mit gewöhnlicher Anilin- und Gramfärbung leicht und intensiv tingierbare hefeähnliche Gebilde. Einzelne besitzen auch ein granuliertes Protoplasma. 
die unter dem Bild der tiefen und oberflächlichen Trichophytie verlief. 235

Beim Hinzufügen von Lauge sieht. man sie am besten (s. Mikrophotogramm Abb. 3). Makroskopisch zeigen die Kolonien in ihrer Jugendform kleinste, mattweise Pünktchen, die sich in den folgenden Tagen mit einem breiten Strahlenkranz umgeben. Die Oberfläche beginnt sich rasch radiär und spiralförmig zu fälteln. Nach einigen Wochen bekommen die Pilzkolonien eine gelbliche, nachher gelbe und schließlich eine bräunliche Verfärbung. Zahlreiche Medien (gewöhnl. Agar, Maltose-, Glykose-, Glycerin-, Gelatine-, Pepton- und Blutserumagar, dann Kartoffel- und Bouillonnährböden) wurden mit

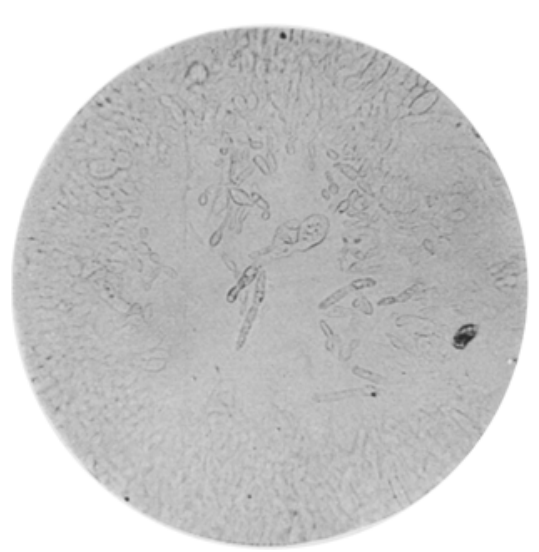

Abb. 3. Zeigt das Auskeimen von Parendo. myces asteroides. 24 Stunden alt, bei $37^{\circ}$ Temp. Verge. $1: 250$.

Zeiss, Immersion $1 / 12$, Ok. 4 .

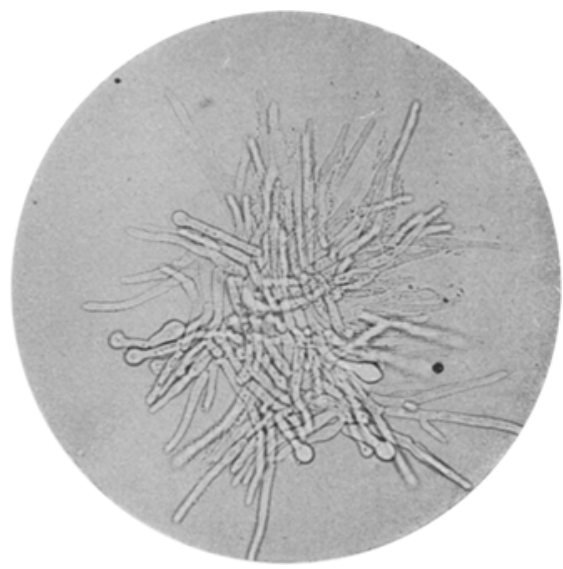

Abb. 4. Die Kultur von Parendomyces asteroides wächst sehr rasch auf zuckerhaltigen Nährböden. Schon in 24 Stunden sieht man ziemlich große Kolonien. Die Mycelien sind verzweigt und zeigen an einzelnen Stellen kolbenartige Auftreibungen. Vergr. 1:250. Zeiss, Immersion 1/12, Ok. 4 .

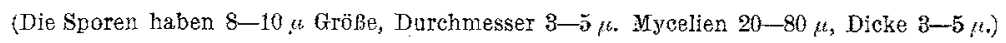

Pilzrasen beschickt und teils bei Zimmertemperatur, teils im Brutofen mehrere Wochen und Monate hindurch beobachtet. Die Pilzkolonien wachsen auf allen Nährböden, bevorzugen aber zuckerhaltige oder mit Gelatine und Kartoffel versetzte Nährböden. Am schlechtesten wachsen sie auf Blutserum- und gewöhnlichem Agar. Bei Zimmertemperatur war das Wachstum üppiger als bei Brutofentemperatur. Rohrzucker, Maltose und Glykose waren sie nicht imstande zu vergären. 9. Tierexperimente. Es wurden mehrere Meerschweinchen, Ratten und Mäuse mit Kulturaufschwemmung percutan, subcutan und intraperitoneal geimpft. Von den geimpften Tieren starb die subcutan geimpfte Maus nach 2 Tagen, die percutan und intraperitoneal geimpften starben nach 3 Tagen. Die Sektion ergab bei der ersten Maus nur eine Milzvergrößerung, bei der zweiten normalen Befund, bei der dritten fanden wir an der Oberfläche der Leber ein hirsekorngroßes; grauweißliches 
Knötchen. Haut und innere Organe o. B. Bei der histologischen Untersuchung zeigte dieses Knötchen zahlreiche Leukocyten und einige den oben erwähnten ähnliche ovoide Gebilde. Es dürfte sich wahrscheinlich bei der dritten Maus bloß um von der Impfung her liegengebliebene Pilzzellen handeln, und der Tod aller drei Mäuse war entweder ein zufälliger (interkurrente Krankheit) oder er trat als Folge einer toxischen Wirkung zerstörter Pilze ein (Dr. Dössekker, Korrespondenzbl. f. Schweiz.

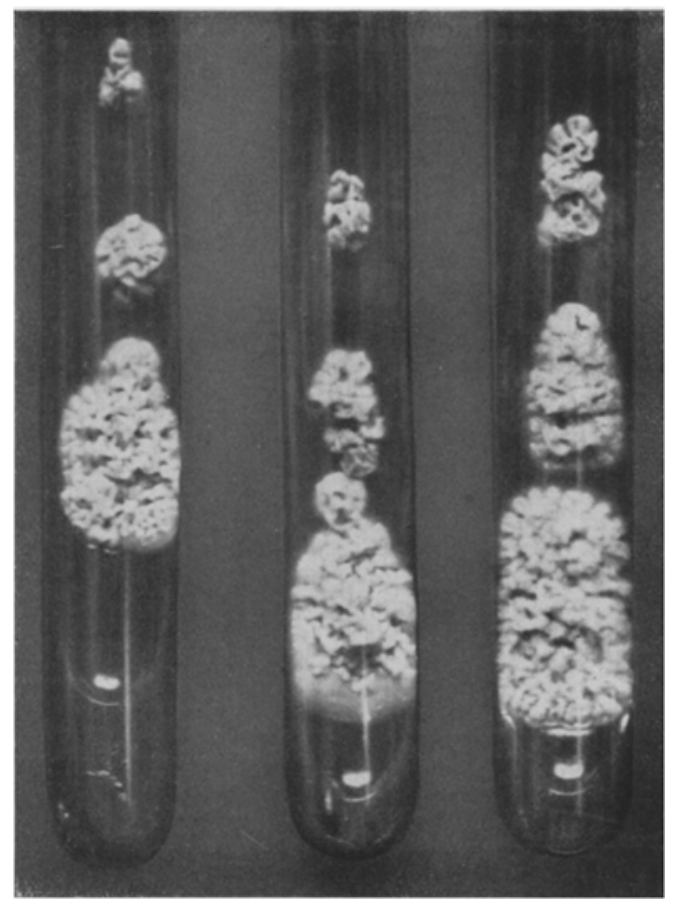

$a$

$c$

Abb. $\check{\circ} . \quad a$ und $b$ Parendomyces asteroides; $c$ Parendomyces Balzeri. Auf Glykose-Agar 28 Tage alt. Ärzte, 1917, Nr. 39). Die übrigen Tiere blieben gesund. Eine Excision eines HautstückesdesPatienten zum Zwecke des Nachweises der Formelemente im Schnitte, sowie der Agglutinationsversuch konnten leider nicht nachgeholt werden.

Die nachher gemeinsam mit Priv.-Dozent Dr. R. Massini vorgenommene Prüfung unseres Stammes führte zu folgenden Ergebnissen. Die nähere Untersuchung, hauptsächlich der Jugendformen, ergab, daß unsere Kultur nicht nur bloß aus hefeähnlichen Zellen, sondern auch aus Hefezellen mit knospigen Mycelien, Endosporen usw. besteht (Abb. 3 u. 4). Zum Vergleich wurden mehrere Stämme von Blastomykose, Saccharomykose und der Parendomyces Balzeri zugezogen. Es wurde eine auffallende Ähnlichkeit mit dem Parendomyces Balzeri sowohl makroskopisch wie mikroskopisch konstatiert. Gleichzeitig haben wir aber auch einen Unterschied zwischen beiden Kulturen feststellen können. Makroskopisch: Balzers Kultur besteht in den ersten Tagen aus kleinen, weißen, warzenartigen Gebilden, die einen schimmernden Glanz besitzen. Unsere Kultur weist am Anfang kleine mattweiße Pünktchen auf, die nebeneinander sitzen und eine zähe Konsistenz haben. Später ragt unsere Kultur stärker empor und besitzt größere Windungen als 
die Balzer-Kultur. Außerdem ist in unserer Kultur die ,Mühlstein". Form, die für Balzers Kolonie charakteristisch ist, nicht ausgesprochen (Abb. 5). Mikroskopisch: in Balzers Kulturen wachsen die Mycelien in einer Richtung, dabei zweigen sich mehrere Mycelien von einem Stammycelium ab. In unserer Kultur wachsen dagegen die Mycelien strahlenartig nach allen Richtungen und zeigen fast keine Neigung, sich weiter zu verzweigen. Die Mikrophotogramme zeigen die Verhältnisse besser, als Worte es beschreiben können. Man könnte unseren Stamm als Par endomyces asteroides bezeichnen, eine Bezeichnung, die seiner Morpho'ogie entsprechen würde (Abb. 6, 7, 8 u. 9).

Bekanntlich wurde die erste Parendomyceserkrankung im Jahre 1912 von Balzer, Gougerot und B urnier unter dem Namen ,Parendomycose gommeuse ulcereuse " beschrieben (Ann. de dermatol. et de syphiligr. 3, 282. 1912). Bei einer Pat. traten im Verlaufe von 2 Jahren nußgroße Knoten an der Außenseite des rechten Oberschenkels auf, die indolent waren und spontan perforierten. Nach Entleerung des glasigserösen Inhaltes sank die wenig infiltrierte Wand der Absceßhöhle in sich zusammen, und um die Fistelöfnung entstand eine kraterförmige Ulceration. Die Geschwürsflächen vernarbten teils, teils bedeckten sie sich mit wuchernden, papillären Granulationen. Die mikroskopische Untersuchung des aus einem fluktuierenden Tumor gewonnenen Eiters zeigte keine sichtbaren Keime. Ver-

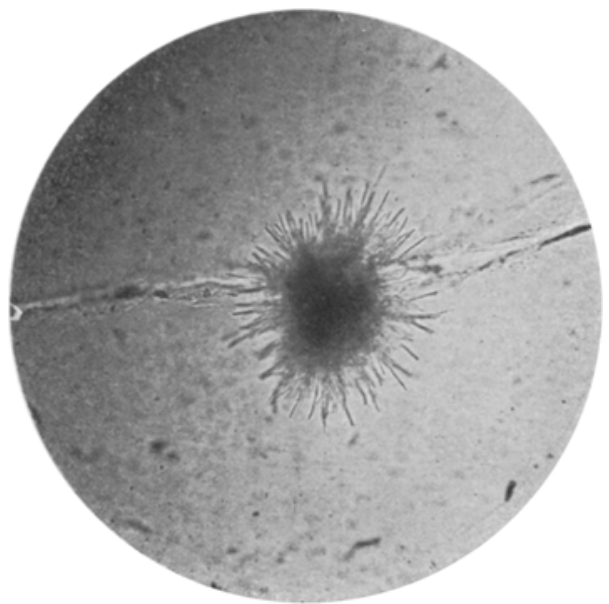

Abb. 6. Parendomyces asteroides. Vergr. $1: 50$. Nach 20 Stunden zeigt sich schon deutlich das sternförm i g e Wachstum. Zeiss, Obj. A, Ok. 4 .

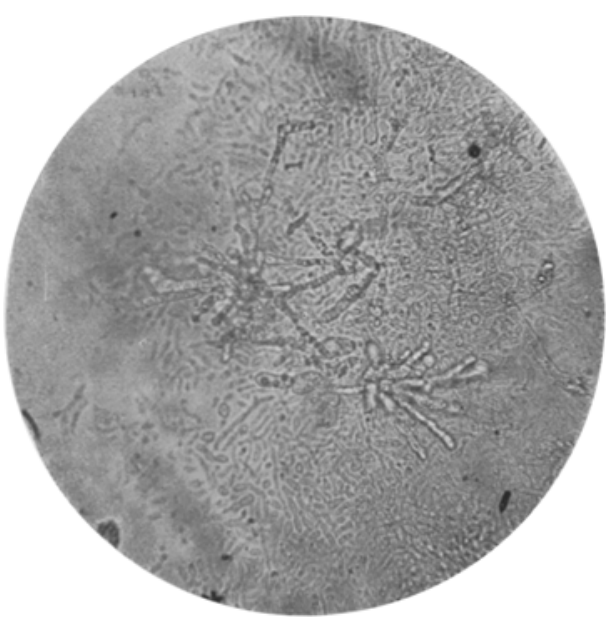

Abb. 7. Vergr. 1:200. Zum Vergleich Parencomyces Balzeri. Die Mycelien beginnen nach 30 Stunden erst auszukeimen. Zeiss, Obj. DD, Ok. 4.

impfung des Eiters ergab nach einigen Tagen spärliche weiße Kolonien eines Hefepilzes. Durch Darreichung von Jodkali heilte diese Affelstion im Laufe eines Monats.

Auf den ersten Blick schien den Autoren (Balzer, Gougerot und Burnier) die von ihnen gezüchtete Kultur der Hefe- oder der Blastomykosegruppe zuzugehören: ,Le champignon isolé par culture appartient au groupe vague et complexe 
des levures ou blastomycètes" (S. 286) und weiter: "le parasite de cette mycose humaine se classe dans le groupe des levures pathogènes" (S. 289). Genauere Untersuchungen ihres Stammes und vergleichende Studien der verschiedenen Pilzarten

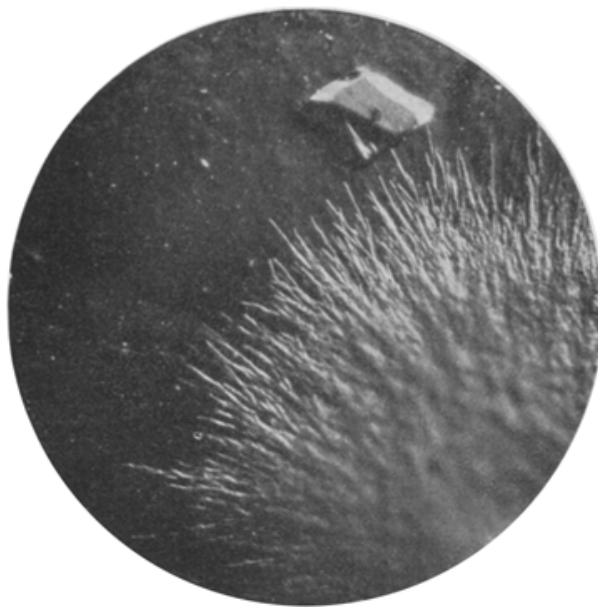

Abb. \&. Vergr. 1:50. Zeigt bei Junkelfeldbeleuchtung die strahlenartige Anordnung einer älteren Kultur von Parendomyces asteroides. Zeiss, Obj. DD, Ok, 4.

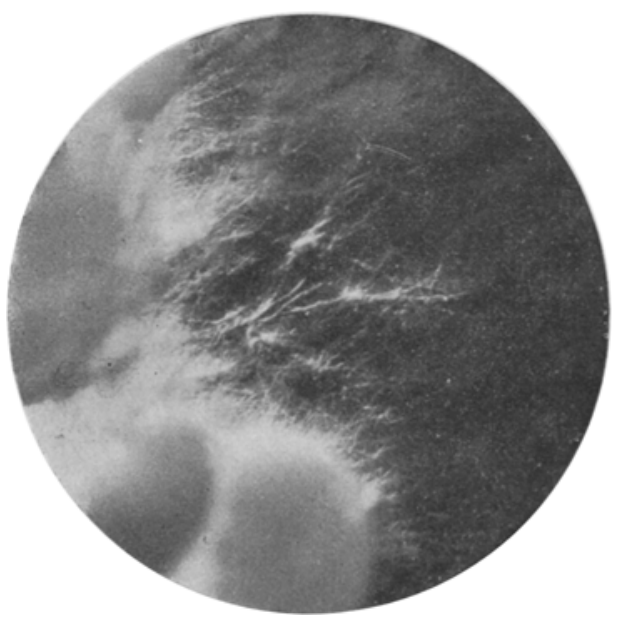

Abb. 9. Vergr. 1:50. Im Vergleich mit Abb. 8 zeigt den mehr baumartig verästelten Rand vor paren. domyces Balzeri. Die Mycelien sind viel feiner als diejenigen des Parendomyces asteroides. Zeiss, Obj. DJ,, $6 \mathrm{k} .4$. Definition „Blastomycètes" genau so unlogisch und künstlich, wie wenn man dic „Bacillose" beschreiben wollte, indem man Diphtherie, Typhus, Rotz, Gangrän, 'Tuberkulose, Tetanus usw. in einer Gruppe vereinigt, unter dem Vorwand, daß alle Erreger dieser Krankheiten eine Bacillusform besitzen. „Aus der Unkenntnis 
dieser einfachen Tatsachen folgt die unglaubliche Verwirrung in der Gruppierung der Blastomyceten und Blastomykose" (S. 292). Diẹse Verwirrung ist auch einem der bedeutendsten Forscher auf diesem Gebiete, A. Busch ke, nicht entgangen, der aber aus „,medizinisch-praktischen Standpunkten" die Bezeichnung Blastomyceten und Blastomykose beibehalten möchte (s. Morphologie und Biologie der Sproßpilze, Kolle u. Wasserman n. 2. Aufl. Bd. 5). In Anbetracht dieser großen Widersprüche und Unklarheiten wurde im Jahre 1909 von Be urmann und Go ugerot (Les Exascoses, Revision et démembrement de l'ancien groupe des blastomycoses. Tribune médicale 1909, zit. nach Balzer) darauf hingewiesen, daß die Bezeichnung Blastomykose und Blastomycetes rom botanischen Standpunkt fallen gelassen werden müsse; sie schlugen ihrerseits für die ganze Gruppe die Bezeichnung Exascose vor und gaben den einzelnen Krankheitsgruppen nach den dabei gefundenen Pilzen auf Grund rein botanischer Gesichtspunkte spezielle Bezeichnungen. Mit einer gewissen Wahrscheinlichkeit wollen sie auch den Parendomyces hinzurechnen (die Bezeichnung Parendomyces, so benannt nach Analogie mit dem Paratyphus, wurde schon von Queyrat und Laroche für einen Parasiten gebraucht, den sie bei der Metrovaginitis pseudomembranacea gefunden haben: den Parendomyces albus). „Das ist nur eine provisorische Einteilung", sagen die obengenannten Autoren, d. h. bis ein genaueres höheres System gefunden wird, das dann die Parasiten in andere, besser bestimmte botanische Gruppen einteilen wird.

Kehren wir jetzt zu unserem Falle zurück, so müssen wir bemerken, daß wir weder unter allen Stämmen von Blastomykose und Saccharomykose, noch unter den Abbildungen, die uns zur Verfügung standen, eine ähnliche Kultur gefunden haben. Bezeichnen wir unsere Kultur mit dem Wort,Blastomykose" im allgemeinen Sinne - so heißt es nach Balzer ,confondre pêle-mêle sous une même etiquette". Sie einfach Parendomyces zu nennen, wäre vielleicht passender als Blastomyces. Und wenn wir noch das Epitheton ,asteroides" hinzufügen, so geschieht dies aus rein morphologischen Gründen.

Sind nun die Züchtungsergebnisse beweiskräftig? Auf diese Frage gibt Bloch in seiner ,Lehre von den Dermatomykosen" folgende Antwort: ,Nicht die Klinik, jedenfalls nicht diese allein, sondern die Züchtung auf künstlichen Nährböden und das Tierexperiment haben hier das entscheidende Wort zu sprechen, so gut wie auf allen übrigen Gebieten der infektiösen und kontagiösen Krankheiten" (Arch. f. Dermatol. u. Syphilis 93, 158. 1908). Die Züchtung der Kulturen ist aber nicht immer leicht. Nach der Statistik von Marzinowsky und Bogrow (also bis 1907) wurden Züchtungsversuche in 45 Fällen von Hautblastomykose und dabei $16 \mathrm{mal}$ erfolglos angestellt. In den übrigen 29 Fällen bekam man die Kultur: 3 mal zweifellose Hefen (Busse - Buschkes Fall), Curtis Fall und Hesslers Fall), in 17 Fällen Schimmelpilze und Oidiumarten, in 9 Fällen unreine Kultur oder die Details nicht angegeben. In 21 Fällen wurden auch Impfungsversuche an Tieren angestellt: $10 \mathrm{mal}$ ohne Erfolg, 11 mal erwies sich die Kultur pathogen. (Arch. f. Dermatol. u. Syphilis 86, 215. 1907).

Wir müssen noch zu der Möglichkeit Stellung nehmen, ob die ge- 
fundenen Parasiten nicht einen zufälligen Befund darstellen. Die akzidentelle Anwesenheit der Hefezellen bei verschiedenen Hautkrankheiten, wie auch bei bösartigen Geschwülsten, scheint nicht ausgeschlossen zu sein. Bekanntlich hat man sie bei verschiedenen Dermatosen (bei Lupus erythematodes, Psoriasis, Alopecia seborrhoica capillitii, Eczema madidans usw. nach Marzinowsky und Bogrow) und anderen Krankheiten (Ribbert und Cernek in Hirnabscessen, Wagner in Muskulatur des Oesophagus und in benachbarten Blutgefäben, Parrot in den Lungen und im Peritoneum, Schmorl in Nierenabscessen) nachgewiesen. Wir glauben aber nicht, daß in unserem Falle von einem zufälligen Befunde gesprochen werden kann, denn dieser Pilz wurde doch bei anderen Hautkrankheiten nicht gefunden; zweitens spricht gegen den zufälligen Befund die große Ähnlichkeit mit dem Parendomyces Balzeri, der als pathogen angesehen wurde. Auch im Fall von Balzer gelang es nicht, den Parasiten im Eiterausstrich festzustellen. Es wäre vielleicht so zu erklären, daß die Parasiten nicht zu allen Zeiten nachweisbar sind. Andererseits scheinen sie gegen Mischinfektionen empfindlich zu sein (W. Pick, Arch. f. Dermatol. u. Syphilis 123, 727. 1916), oder infolge der Mischinfektion werden sie wahrscheinlich vermißt (O. Stein, Die Gilchristsche Krankheit, Arch. f. Dermatol. u. Syphilis 120, 882. 1914).

\section{Zur Diagnose.}

Der Kranke machte die Angabe, er habe sich von seinen Kälbern infiziert, die an einer Hautaffektion litten. Wir haben nicht die Gelegenheit gehabt, die Tiere zu besichtigen. In solchen Fällen sind aber die anamnestischen Angaben nicht so ohne weiteres von der Hand zu weisen; denn es ist nicht zu leugnen, daß die Beobachtungsgabe der landwirtschafttreibenden Bevölkerung in dieser Hinsicht nicht schlecht entwickelt ist. Die Annahme lag für uns nahe, daß es sich bei der Erkrankung der unbehaarten Körperstellen um eine trichophytieartige Affektion handelte, obwohl der Pilznachweis nicht gelungen war. Das klinisch-morphologische Bild und der Verlauf der Affektion bestärkten uns in dieser Auffassung. Dabei war natürlich zuerst die Frage offen zu lassen, ob diese Trichophytie durch eine eigentliche Trichophytiespezies bedingt sei, oder ob ein anderer Erreger die gleiche ,réaction cutanée" (nach Brocq) ausgelöst habe. Was das Exanthem in der Bartgegend angeht, konnten wir maligne Tumoren, Tuberkulose und Lues ausschließen. Gegen Epitheliom und Tuberkulose sprachen das akute Auftreten der Krankheit, der Verlauf und der Erfolg der Joddarreichung (gegen Tuberkulose auch der negative Ausfall der Tuberkulinreaktionen). Gegen Lues sprach die glatt negative WaR., was im manifesten III. Stadium eine Seltenheit wäre. Größere 
die unter dem Bild der tiefen und oberflächlichen Trichophytie verlief. 241

Schwierigkeiten entstanden bei Beantwortung der Frage, welche Spezies einer tiefen Dermatomykose hier vorlag. Sporotrichose machte der negative Ausfall der Sporotrichinreaktion unwahrscheinlich, ebenso das Fehlen des sonst häufigen Polymorphismus der Läsionen und der charakteristischen meist vorhandenen schleimig-weißlichen Suppurationen, sowie eines Primäraffektes mit lymphangitischen Veränderungen. Auch Aktinomykose kam kaum in Betracht wegen der weichen Konsistenz des erkrankten Gewebes und der Abwesenheit von Actinomycesdrusen. Hinsichtlich anderer differentialdiagnostisch $\mathrm{zu}$ berücksichtigender seltener Dermatomykosen (Discomykose von Rava utPinoy, Hemisporose von Gougerot und Caraven, Oidiomycosis gummosa von Beurmann-Gouggerot-Vaucher, Acremoniose von Potron und Noisette, Kladiose von Bloch und Vischer, Dermatomycosis vegetans von Balzer-Gougerot-Burnier usw.), verweisen wir auf das Ergebnis des Kulturverfuhrens, das keine analogen Verhältnisse zutage förderte. Per exclusionem blieben uns von bekannten Dermatomykosen nur zwei zur weiteren diagnostischen Prüfung, nämlich die Sycosis parasitaria und die Blastomykose ${ }^{1}$ ) übrig. In der 'Schweiz wird die Sycosis parasitaria meistens durch eine Gypseumform hervorgerufen. Die anamnestischen Angaben des Patienten weisen auf diese Möglichkeit hin, da der Kranke selbst meinte, die Ansteckung sei von einem kranken Rinde ausgegangen. Indes fiel uns auf, daß die bei uns übliche und zuerst über 6 Wochen durchgeführte Therapie (feuchte Umsohläge und Röntgen) vollkommen versagte, während wir sonst damit, resp. infolge der fortschreitenden Immunisierungsvorgänge, durchwegs befriedigende Resultate bei der Sycosis parasitaria verzeichneten. Dieser Erfolg wurde schon früher von Jadassohn auf der Berner Klinik beobachtet (Jadassohn, Utber die Trichophytien, Berl. klin. Wochenschr. Nr. 21. 1918). Ein Trichophytiepilz wurde trotz mehrmaliger, mikroskopischer und kultureller Untersuchungen nie gefunden. Dieses negative Ergebnis hat für uns einen gewissen Wert; denn sonst gelingt der Pilznachweis sowohl mikroskopisch als kulturell in der Mehrzahl der Fälle. Wir durften also einen Trichophytonpilz als ätiologischen Faktor wohl ausschließen. Auf Grund der obigen Ausführungen kamen wir zu dem Schlusse, daß die beschriebene Affektion als eine Form der Blastomykose (wir sprechen da von der Blastomykose im engeren Sinne, d. h. vom Typus Busse-Buschke und Gilchrist) oder eine diesem Leiden nahestehende Erkrankung anzusprechen sei, die morphologisch dem Bilde der tiefen Trichophytie (Sycosis parasitaria) in der Bartgegend und an den nicht behaarten Körperstellen der superfiziellen und tiefen Trichophytie entsprach.

1) Nach de Beurmann und Gougerot würde man richtiger von Exascose sprechen.

Archiv f. Dermatologie u. Syphilis. o. Bd. 134. 
Denn es liegt doch am nächsten, die Affektion des Bartes und des übrigen Integumentes als ein und dieselbe Krankheit aufzufassen. Gegen Blastomykose (Typus Busse-Buschke) sprach die Abwesenheit der Knochen- und Gelenkläsionen, der disseminierten gummösen Knötchen, die sich nachher in Ulcera verwandeln, das gute Allgemeinbefinden und die rapide Heilung. Blastomykose vom Typus Gilchrist machte die Seltenheit dieser Krankheit in Europa unwahrscheinlich, ebenso das akute Auftreten, die Abwesenheit der miliaren Abscesse und der Entwicklung einer Affektion, welche derjenigen bei Tuberculosis cutis verrucosa ähnlich ist (A. Buschke, Über die unter der Bezeichnung Hautblastomykose zusammengefaßten Affektionen, Mračeks Handbuch der Hautkrankheiten 4, 2. Hälfte, S. 445 und O. Busse, Die Sproßpilze, Kolle und Wassermann, Bc. 1). Schließlich zeigten die Kulturen, daß wesentliche Verschiedenheiten zwischen den Kulturen der beiden Arten der Blastomykose und der von uns beschriebenen Affektion hervorzuheben sind, so daß es nicht angeht, unser Krankheitsbild mit der Blastomykose $\mathrm{zu}$ identifizieren. An dieser Auffassung kann die Tatsache nichts ändern, daß der Effekt der Joddarreichung ein ebenso auffallender war, wie es uns bekannt ist von der Blastomykose, Aktinomykose und Sporotrichose. Über die Wirkung des Jodkaliums bei tiefen Trichophytien (Sycosis parasitaria und Kerion Celsi) wird seinerzeit Herr Prof. Naegeli ausführlich berichten. Mit Rücksicht auf die Ähnlichkeit der von uns gezüchteten Pilze mit demjenigen Balzers und wegen des in beiden Fällen eklatanten Erfolges der Therapie ist es angezeigt endlich noch die Frage zu beantworten, ob unsere Beobachtung nicht der ,Parendomycose gommeuse ulcereuse" angehöre. Dagegen sprach aber das akute Auftreten, die rasche Ausbreitung des Exanthems und das Fehlen geschwüriger Prozesse. Es scheint sich also $\mathbf{u m}$ ein Krankheitsbild $\mathrm{zu}$ handeln, das klinisch und in seinem Verlaufe der tiefen (Sycosis parasitaria) und oberflächlichen Trichophytie am nächsten steht, ätiologisch indes durch eine bisher noch nicht beschriebene Pilzart bedingt ist, die wir mit dem Namen Parendomyces asteroides belegen. 